\title{
Metastatic Breast Carcinoma to The Uterine Cervix - An Unusual Presentation
}

\author{
Maymol P Varghese*, Sreeja Raju, Savithri M C, Joy Augustine and Mathew Varghese \\ Amala Institute of Medical Sciences, Thrissur
}

\begin{abstract}
Uterine cervix involvement as a site of metastasis from a distant primary tumor is rare. Metastasis from breast carcinoma in a patient undergoing treatment is even rarer. The present case report is of a 47-year-old lady with history of carcinoma breast on treatment and who was referred to our hospital with complaints of lower limb oedema, low backache and postmenopausal whitish discharge per vaginum. Per vaginal examination revealed a lesion arising in the cervix extending up to the anterior vaginal wall suggestive of a clinical diagnosis of carcinoma cervix. Subsequent biopsy from the lesion showed malignant cells arranged in Indian file pattern and focal alveolar pattern, hinting at a diagnosis of Invasive lobular carcinoma metastasis. The neoplasm was positive for ER, PR and GATA-3 and negative for Her2/neu and E-cadherin confirming our diagnosis. Although rare, the poor outcome in such patients makes it even more important for its identification and the need for them to undergo careful routine gynecologic examination.
\end{abstract}

Keywords: Lobular Carcinoma, Uterine Cervix. E-cadherin

\section{Introduction}

Uterine cervix involvement as a site of metastasis from a distant primary tumor is rare. Metastasis from breast carcinoma in a patient undergoing treatment is even rarer. Here we present the case of a 47-year-old lady on tamoxifen treatment for carcinoma breast presenting with postmenopausal discharge. Clinical diagnosis was that of carcinoma cervix. Correct diagnosis is imperative as the management for primary genital tract carcinoma and metastatic breast carcinoma are dramatically different.

\section{Case Report}

47-year-old lady was referred to our hospital with complaints of lower limb oedema, low backache and postmenopausal whitish discharge per vaginum. 6 years previously, she has undergone left breast lump excision which suggested lobular carcinoma breast. Tumor was positive for ER and PR and was negative for Her 2. She further underwent Modified radical mastectomy with node dissection. Postoperative histological examination showed no evidence of residual tumor. Out of 14 lymph nodes dissected seven were found to be positive. Subsequently she received 3 cycles of Adriamycin with cyclophosphamide and 3 cycles of paclitaxel followed by adjuvant radiotherapy. She was started on tamoxifen and was under regular follow up without any recurrence. She also gives a family history of carcinoma breast and endometrium in first degree relative.

Physical examination during her presentation revealed bilateral pedal oedema. There was no pallor or lymphadenopathy. The right breast and axilla were normal. There were no signs of local recurrence. No other significant findings were observed during her abdominal examination. Pelvic examination revealed a hard mass arising in the cervix extending up to the anterior vaginal wall. A clinical diagnosis of carcinoma cervix was considered.

CT examination revealed bulky uterus and cervix. Cervix showed heterogenous enhancement. Serosal irregularity with parametrial fat stranding was also noted. No other metastatic foci were identified.

Biopsy from the lesion in the cervix and vagina was done and it revealed malignant cells $\mathrm{n}$ arranged in Indian file pattern and focal alveolar pattern hinting at a possible metastasis from lobular carcinoma breast. [Figure 1]. Endometrium was atrophic. Immunohistochemical [IHC] studies showed tumor positivity for GATA-3 [Figure 2] confirming metastatic deposits from breast. ER [Fig 3] and PR was positive. Her2/neu and E-cadherin [Figure 4] were negative in the tumor cells. PAX 8 was negative ruling out primary of female genital system. [Figure 5]. Immunomorphology was consistent with lobular carcinoma metastasis to cervix. Palliative chemotherapy with 3 cycles of paclitaxel and carboplatin was initiated.

\section{Discussion}

Metastases in the female genital tract from an extra genital primary tumour though uncommon is seen in ovaries and vagina. Uterine cervix involvement is very rare owing to its limited blood supply and afferent lymphatic drainage. 


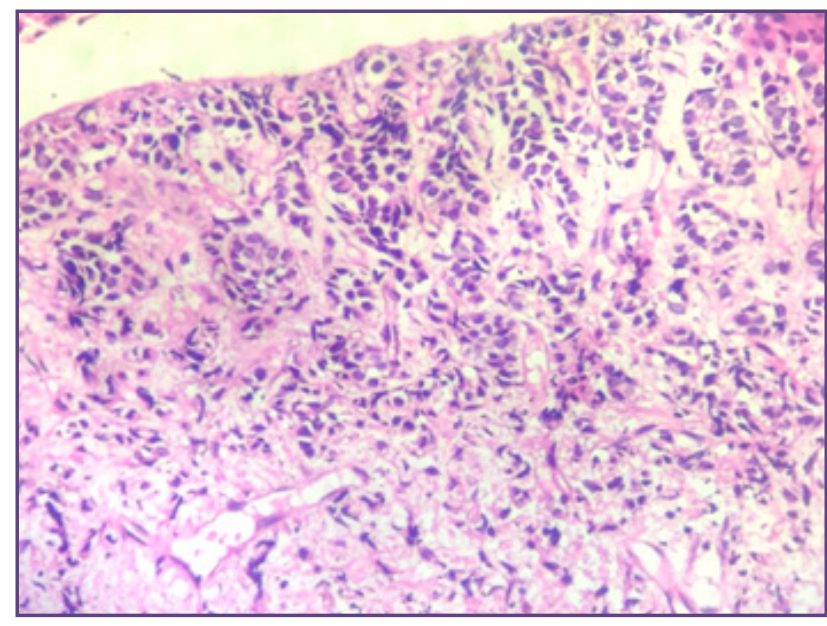

Fig. 1: Biopsy from cervix and vagina showed malignant cell infiltration arranged in Indian file pattern and focal alveolar pattern.

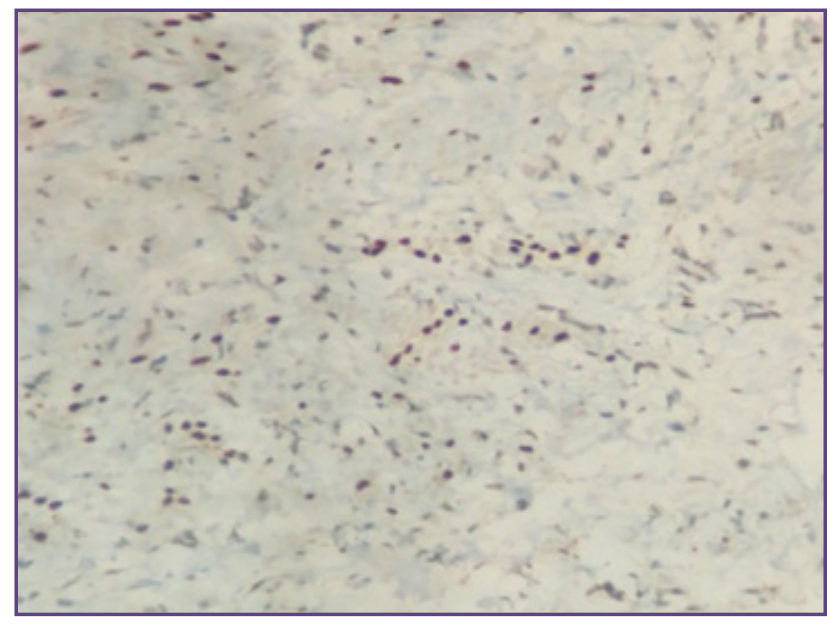

Fig. 3: ER positive in tumor.

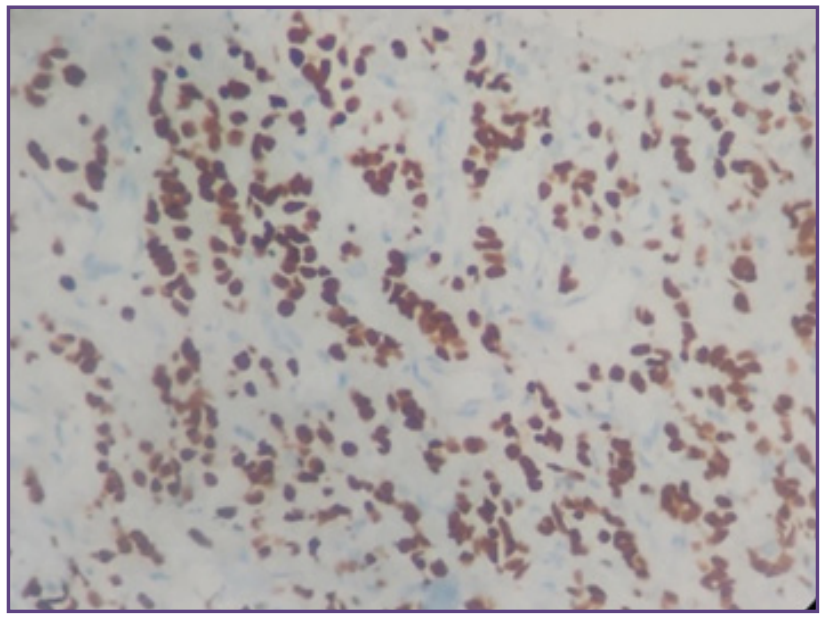

Fig. 2: GATA 3 - positive in tumor cells.

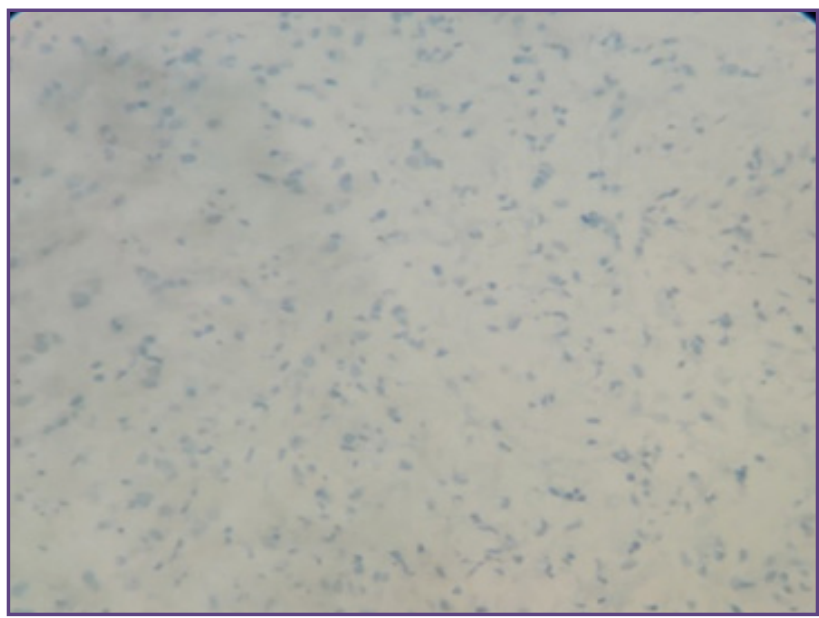

Fig. 4: E-cadherin negative in tumor cells.



Fig. 5: PAX8 negative in tumors cells. 
Most common tumour metastasing to cervix are from gastrointestinal tract and ovarian cancers. ${ }^{[1]}$ Metastatic deposits from breast cancer to cervix uteri is a rare phenomenon.

Among the breast carcinoma subtypes, lobular histologic type seems to metastasise to the genital tract more frequently. Other unusual metastatic sites of lobular carcinoma include bones, peritoneum, retroperitoneum and gastrointestinal tract. ${ }^{[2]}$ This unique ability to metastasize to distant organs is attributable to the inactivation of Cadherin 1 [CDH1] gene at $16 \mathrm{q} 22$ which leads to loss of E cadherin expression. This leads to abnormalities in catenin expression which results in loss of cell-to-cell adhesion and intracellular and intercellular signalling promoting invasion and metastasis.

[3] Radiologically lobular carcinomas are more difficult to detect in breast as well as at metastatic sites. This is attributable to the fact that lobular tumours tend to grow as sheets of cancer cells rather than discrete masses and induce less desmoplastic reaction. ${ }^{[4]}$ Microscopically, metastases from lobular carcinoma consist of tumour cells arranged in an Indian file pattern. The IHC markers for ILC are Gross cystic disease fluid protein -15 [GCDFP -15], loss of E Cadherin and positivity for ER and PR. ${ }^{[5]}$ Other markers suggestive of metastasis are GATA 3 and mammaglobin.

Correct diagnosis is imperative as the management for primary genital tract carcinoma and metastatic lobular carcinoma are dramatically different.

\section{Conclusion}

Correct diagnosis is imperative as the management for primary genital tract carcinoma and metastatic lobular carcinoma are dramatically different.

\section{Acknowledgements}

None

\section{Funding}

None

\section{Competing Interests}

None Declared

\section{Reference}

1. Pérez-Montiel D et al; Adenocarcinoma metastatic to the uterine cervix: a case series J Obstet Gynaecol Res.2012 ;38(3) 541-9

2. Cao AY et al; Tumor characteristics and the clinical outcome of invasive lobular carcinoma compared to infiltrating ductal carcinoma in a Chinese Population World J Surg Oncol 2012

3. Engelstaedter $\mathrm{V}$ and Mylonas I ; Lower genital tract metastases at time of first diagnosis of mammary invasive lobular carcinoma ;Arch Gynecol Obstet 2011;283(1) 93-5

4. Rajitha L et al;Metastatic lobular carcinoma of breast mimics primary cervix carcinoma: two case reports and a review of the literature. ecancer 2015;9:571

5. De Leeuw WJ, Berx G et al: Simultaneous loss of E-cadherin and catenins in invasive lobular breast cancer and lobular carcinoma in situ: J Pathol (1997);183:404-411.

*Corresponding author:

Maymol P Varghese, Amala Institute of Medical Sciences, Thrissur

Phone: +91 9961256704

Email: may.ann181@gmail.com

Date of Submission $\quad: 08 / 10 / 2020$

Date of Final Revision : 20/01/2021

Date of Acceptance $\quad: 26 / 01 / 2021$

Financial or other Competing Interests: None.

Date of Publication $\quad: 28 / 02 / 2021$ 